\title{
EXACTNESS OF LEPAGE 2-FORMS AND GLOBALLY VARIATIONAL DIFFERENTIAL EQUATIONS
}

\author{
ZBYNĚK URBAN AND JANA VOLNÁ
}

\begin{abstract}
The exactness equation for Lepage 2-forms, associated with variational systems of ordinary differential equations on smooth manifolds, is analyzed with the aim to construct a concrete global variational principle. It is shown that locally variational systems defined by homogeneous functions of degree $c \neq 0,1$ are automatically globally variational. A new constructive method of finding a global Lagrangian is described for these systems, which include for instance the geodesic equations in Riemann and Finsler geometry.
\end{abstract}

\section{INTRODUCTION}

Our aim is to study a construction of a global Lagrangian for globally variational equations on fibered tangent bundles of smooth $m$-dimensional manifolds. In our recent work [17, we gave a solution to this problem for $m=2$ by means of applying the de Rham top-cohomology theory; in part we now generalize the methods of 17] to dimension $m \geq 2$. In general, however, the problem remains open since we proceeded in this paper for a class of homogeneous differential equations of degree $c \neq 0,1$ only. The topic belongs to studies of the influence of topology on variationality of differential equations, and on the existence and a construction of the corresponding local and global variational principles; see Krupka, Urban and Volná [7.

The existence of a global variational principle for (ordinary or partial) differential equations is influenced by the topology of the underlying space. For ordinary variational equations of arbitrary order it depends on the second de Rham cohomology group $H_{\mathrm{dR}}^{2} Y$ of the underlying fibered manifold $Y$ : if $H_{\mathrm{dR}}^{2} Y$ is trivial, then a locally variational source form $\varepsilon$ on the $r$-th jet prolongation $J^{r} Y$ is also globally variational. This important result is due to Takens [13] (see also Krupka [2], and for further comments Krupka et al. [6]), is, however, sheaf-theoretic and to the authors' knowledge there is no general method how to construct a global Lagrangian for locally variational equations. Simple examples also show that the well-known Vainberg-Tonti formula (cf. Tonti [19]) fails to produce global Lagrangians.

The main idea is based on solvability of the global exactness equation for the Lepage equivalent $\alpha_{\varepsilon}$ of a variational source form $\varepsilon$, associated with a given system of variational second-order ordinary differential equations (cf. Krupka [5], Krupková and Prince [10]). Globally defined 2-form $\alpha_{\varepsilon}$ on $\mathbb{R} \times T M$ represents an example of a Lepage 2-form in Lagrangian mechanics (see Krupková [8]), satisfying the equation $\alpha_{\varepsilon}=d \Theta_{\lambda}$, where $\Theta_{\lambda}$ is the well-known Cartan form, which depends on the

2010 Mathematics Subject Classification. 58A15; 58E30; 34A26; 53C22.

Key words and phrases. Variational differential equation; Lagrangian; Euler-Lagrange expressions; Helmholtz conditions; Lepage form; homogeneous function.

ZU appreciates support of the Visegrad grant No. 51810810 at the University of Prešov. 
choice of a Lagrangian $\lambda$ whereas $d \Theta_{\lambda}$ does not. As a result, we reduce the global exactness of the Lepage equivalent $\alpha_{\varepsilon}$ of $\varepsilon$ to global exactness of a certain 2-form globally defined on $M$ (cf. Theorems 8 and 9). Apparently, the topology of $M$ decides on global exactness of $\alpha_{\varepsilon}$. The meaning of Lepage forms for the calculus of variations and their basic properties have been reviewed by Krupka, Krupková and Saunders [3].

In the class of second-order ordinary differential equations, given by variational and homogeneous of degree $c \neq 0,1$ functions, we describe a new construction of a global variational principle, which does not depend on the topology of the underlying manifold. We prove that locally variational source forms with homogeneous coefficients of degree $c \neq 0,1$ are automatically globally variational, and possess a global Lagrangian given by Theorem 14. Note that systems of second-order positive-homogenous (of degree 1) differential equations, characterized by the wellknown Zermelo conditions, were also studied from the variational point of view by Urban and Krupka [15, 16.

For notation and basic geometric structures well adapted to this work, we refer to Krupka, Urban, and Volná [7]. Throughout, we consider fibered manifolds which are the Cartesian products $Y=\mathbb{R} \times M$ over the real line $\mathbb{R}$ and projection $\pi$ : $\mathbb{R} \times M \rightarrow \mathbb{R}$, where $M$ is a general smooth manifold of $\operatorname{dimension} \operatorname{dim} M=m \geq 2$. Clearly, the jet spaces $J^{1} Y$ and $J^{2} Y$ can be canonically identified with products $\mathbb{R} \times T M$ and $\mathbb{R} \times T^{2} M$, respectively, where $T M$ is the tangent bundle of $M$, and $T^{2} M$ denotes the manifold of second-order velocities over $M$. The canonical jet projections are denoted by $\pi^{2}: \mathbb{R} \times T^{2} M \rightarrow \mathbb{R}, \pi^{2,0}: \mathbb{R} \times T^{2} M \rightarrow \mathbb{R} \times M$, and $\pi^{2,1}: \mathbb{R} \times T^{2} M \rightarrow \mathbb{R} \times T M$. Recall that elements of $T^{2} M$ are 2 -jets $J_{0}^{2} \zeta \in J^{2}(\mathbb{R}, M)$ with origin $0 \in \mathbb{R}$ and target $\zeta(0) \in M$. These jet spaces are endowed with the natural fibered manifold structure: if $(V, \psi), \psi=\left(x^{i}\right)$, is a chart on $M$, then $\left(\mathbb{R} \times V, \operatorname{id}_{\mathbb{R}} \times \psi\right), \operatorname{id}_{\mathbb{R}} \times \psi=\left(t, x^{i}\right)$, is a fibered chart on $\mathbb{R} \times M$, and the associated charts on $\mathbb{R} \times T M$ and $\mathbb{R} \times T^{2} M$ reads $\left(\mathbb{R} \times V^{1}, \operatorname{id}_{\mathbb{R}} \times \psi^{1}\right), \operatorname{id}_{\mathbb{R}} \times \psi^{1}=\left(t, x^{i}, \dot{x}^{i}\right)$, and $\left(\mathbb{R} \times V^{2}, \operatorname{id}_{\mathbb{R}} \times \psi^{2}\right), \operatorname{id}_{\mathbb{R}} \times \psi^{2}=\left(t, x^{i}, \dot{x}^{i}, \ddot{x}^{i}\right)$, respectively. Here $V^{1}$ and $V^{2}$ are preimages of $V$ in the canonical tangent bundles projections $T M \rightarrow M$ and $T^{2} M \rightarrow M$.

The exterior algebra of differential forms on $\mathbb{R} \times T M$, resp. $\mathbb{R} \times T^{2} M$, is denoted by $\Omega^{1}(\mathbb{R} \times M)$, resp. $\Omega^{2}(\mathbb{R} \times M)$. By means of charts, we put $h d t=d t, h d x^{i}=$ $\dot{x}^{i} d t, h d \dot{x}^{i}=\ddot{x}^{i} d t$, and for any function $f: \mathbb{R} \times T M \rightarrow \mathbb{R}, h f=f \circ \pi^{2,1}$. These formulas define a global homomorphism of exterior algebras $h: \Omega^{1}(\mathbb{R} \times M) \rightarrow$ $\Omega^{2}(\mathbb{R} \times M)$, called the $\pi$-horizontalization. A 1 -form $\rho \in \Omega^{1}(\mathbb{R} \times M)$ is called contact, if $h \rho=0$. With respect to a chart $(V, \psi), \psi=\left(x^{i}\right)$, on $M$, every contact 1 -form $\rho$ has an expression $\rho=A_{i} \omega^{i}$, for some functions $A_{i}: \mathbb{R} \times V^{1} \rightarrow \mathbb{R}$, where $\omega^{i}=d x^{i}-\dot{x}^{i} d t$. For any differential 1 -form $\rho \in \Omega_{1}^{1}(\mathbb{R} \times M)$, the pull-back $\left(\pi^{2,1}\right)^{*} \rho$ has a unique decomposition $\left(\pi^{2,1}\right)^{*} \rho=h \rho+p \rho$, where $h \rho$, resp. $p \rho$, is $\pi^{2}$-horizontal (respectively, contact) 1 -form on $\mathbb{R} \times T^{2} M$. This decomposition can be directly generalized to arbitrary $k$-forms. For $k=2$, if $\rho \in \Omega_{2}^{1}(\mathbb{R} \times M)$ is a 2 -form on $\mathbb{R} \times T M$, then we get $\left(\pi^{2,1}\right)^{*} \rho=p_{1} \rho+p_{2} \rho$, where $p_{1} \rho$ (resp. $p_{2} \rho$ ) is the 1 -contact (respectively, 2-contact) component of $\rho$, spanned by $\omega^{i} \wedge d t$, (respectively, $\left.\omega^{i} \wedge \omega^{j}\right)$. Analogously, we employ these concepts on $\mathbb{R} \times T^{2} M$.

The results of this work can be generalized to higher-order variational differential equations by means of similar methods. Another non-trivial extension consists in 
replacing Cartesian product $\mathbb{R} \times M$ by a general fibered manifold over 1-dimensional base, and extension to partial differential equations.

\section{ORDinARY VARIATIONAL EQUATIONS AND LEPAGE 2-FORMS}

In coherence with the general theory of ordinary variational differential equations on fibered spaces (cf. Krupková and Prince [10], and references therein), we give basic definitions and concepts, adapted to our underlying structures.

Let $\varepsilon$ be a source form on $\mathbb{R} \times T^{2} M$, i.e. $\pi^{2,0}$-horizontal 1-contact 2-form, locally expressed as

$$
\varepsilon=\varepsilon_{i} \omega^{i} \wedge d t
$$

with respect to a chart $(V, \psi), \psi=\left(x^{i}\right)$, on $M$. In (2.1), we suppose the coefficients $\varepsilon_{i}, 1 \leq i \leq m$, are differentiable functions on $V^{2} \subset T^{2} M$, and $\omega^{i}=d x^{i}-\dot{x}^{i} d t$, are contact 1 -forms on $\mathbb{R} \times V^{1}$. These assumptions mean that we restrict ourselves to autonomous systems of second-order differential equations, defined by functions

$$
\varepsilon_{i}\left(x^{j}, \dot{x}^{j}, \ddot{x}^{j}\right)=0,
$$

for unknown differentiable curves $\zeta$ in $M, I \ni t \rightarrow \zeta(t)=\left(x^{j}(\zeta(t))\right)$ on an open interval $I \subset \mathbb{R}$.

Source form $\varepsilon$ (2.1) (or system (2.2)) is called locally variational, if there exists a real-valued function $\mathscr{L}: \mathbb{R} \times V^{2} \rightarrow \mathbb{R}$ such that system (2.2) coincide with the Euler-Lagrange equations associated with $\mathscr{L}$, that is, $\varepsilon_{i}=E_{i}(\mathscr{L})$ are the EulerLagrange expressions of $\mathscr{L}$,

$$
E_{i}(\mathscr{L})=\frac{\partial \mathscr{L}}{\partial x^{i}}-\frac{d}{d t} \frac{\partial \mathscr{L}}{\partial \dot{x}^{i}}+\frac{d^{2}}{d t^{2}} \frac{\partial \mathscr{L}}{\partial \ddot{x}^{i}} .
$$

$\mathscr{L}$ is called a (local) Lagrange function for $\varepsilon$. By a Lagrangian for fibered manifold $\mathbb{R} \times M$ over $\mathbb{R}$ we call a $\pi^{2}$-horizontal 1 -form $\lambda$ on an open subset $W^{2} \subset \mathbb{R} \times T^{2} M$; in a fibered chart we have $\lambda=\mathscr{L}\left(t, x^{i}, \dot{x}^{i}, \ddot{x}^{i}\right) d t$. The mapping $\lambda \rightarrow E_{\lambda}$, assigning to a Lagrangian $\lambda$ the Euler-Lagrange form $E_{\lambda}$, is the well-known Euler-Lagrange mapping in the calculus of variations; in a fibered chart we have

$$
E_{\lambda}=E_{i}(\mathscr{L}) \omega^{i} \wedge d t
$$

We remark that a Lagrangian represents a class of 1-forms, and a source form represents a class of 2-forms in the (quotient) variational sequence over $W$ (see Krupka [2, and also Krupka et al. 4]), in which the Euler-Lagrange mapping is one of its morphisms.

The coefficients of a locally variational source form $\varepsilon$ (2.1) coincide with the Euler-Lagrange expressions of a Lagrange function with respect to every fibered chart. Note also that such a Lagrange function can always be reduced to an equivalent first-order Lagrange function $\mathscr{L}=\mathscr{L}\left(t, x^{i}, \dot{x}^{i}\right)$ for $\varepsilon$. Local Lagrange functions for $\varepsilon$, defined on chart neighborhoods in $\mathbb{R} \times T M$, need not define a global Lagrange function for $\varepsilon$ on $\mathbb{R} \times T M$. If there exists a Lagrange function $\mathscr{L}$ for $\varepsilon$, defined on $\mathbb{R} \times T M$, we call $\varepsilon$ globally variational.

The following theorem summarizes necessary and sufficient conditions for local variationality of $\varepsilon$.

Theorem 1. Let $\varepsilon$ be a source form on $\mathbb{R} \times T^{2} M$, locally expressed by (2.1) with respect to a chart $(V, \psi), \psi=\left(x^{i}\right)$, on $M$. The following conditions are equivalent:

(a) $\varepsilon$ is locally variational. 
(b) Functions $\varepsilon_{i}, 1 \leq i \leq m$, satisfy the following system identically,

$$
\begin{aligned}
& \frac{\partial \varepsilon_{i}}{\partial \ddot{x}^{j}}-\frac{\partial \varepsilon_{j}}{\partial \ddot{x}^{i}}=0 \\
& \frac{\partial \varepsilon_{i}}{\partial \dot{x}^{j}}+\frac{\partial \varepsilon_{j}}{\partial \dot{x}^{i}}-\frac{d}{d t}\left(\frac{\partial \varepsilon_{i}}{\partial \ddot{x}^{j}}+\frac{\partial \varepsilon_{j}}{\partial \ddot{x}^{i}}\right)=0, \\
& \frac{\partial \varepsilon_{i}}{\partial x^{j}}-\frac{\partial \varepsilon_{j}}{\partial x^{i}}-\frac{1}{2} \frac{d}{d t}\left(\frac{\partial \varepsilon_{i}}{\partial \dot{x}^{j}}-\frac{\partial \varepsilon_{j}}{\partial \dot{x}^{i}}\right)=0 .
\end{aligned}
$$

(c) Functions $\varepsilon_{i}, 1 \leq i \leq m$, are linear in the second derivatives, i.e.

$$
\varepsilon_{i}=A_{i}+B_{i j} \ddot{x}^{j}
$$

and the functions $A_{i}, B_{i j}, 1 \leq i, j \leq m$, depend on $x^{i}, \dot{x}^{i}$ only, and satisfy the conditions,

$$
\begin{aligned}
& B_{i j}=B_{j i}, \quad \frac{\partial B_{i k}}{\partial \dot{x}^{j}}=\frac{\partial B_{j k}}{\partial \dot{x}^{i}}, \\
& \frac{\partial A_{i}}{\partial \dot{x}^{j}}+\frac{\partial A_{j}}{\partial \dot{x}^{i}}-2 \frac{\partial B_{i j}}{\partial x^{k}} \dot{x}^{k}=0, \\
& \frac{\partial A_{i}}{\partial x^{j}}-\frac{\partial A_{j}}{\partial x^{i}}-\frac{1}{2} \frac{\partial}{\partial x^{k}}\left(\frac{\partial A_{i}}{\partial \dot{x}^{j}}-\frac{\partial A_{j}}{\partial \dot{x}^{i}}\right) \dot{x}^{k}=0 .
\end{aligned}
$$

(d) The function

$$
\mathscr{L}=\mathscr{L}_{T}-\frac{d}{d t}\left(x^{i} \int_{0}^{1} C_{i}\left(s x^{k}, s \dot{x}^{k}\right) d s\right),
$$

where functions $C_{i}$ are given by $B_{i j}=\partial C_{i} / \partial \dot{x}^{j}=\partial C_{j} / \partial \dot{x}^{i}$, and

$$
\mathscr{L}_{T}=x^{i} \int_{0}^{1} \varepsilon_{i}\left(s x^{k}, s \dot{x}^{k}, s \ddot{x}^{k}\right) d s
$$

is a Lagrange function for $\varepsilon$ defined on $V^{1}$.

(e) To every point of $\mathbb{R} \times T^{2} M$ there is a neighborhood $W$ and a 2 -contact 2-form $F_{W}$ on $W$ such that the form $\alpha_{W}=\left.\varepsilon\right|_{W}+F_{W}$ is closed.

(f) There exists a closed 2 -form $\alpha_{\varepsilon}$ on $\mathbb{R} \times T M$ such that $\varepsilon=p_{1} \alpha_{\varepsilon}$. If $\alpha_{\varepsilon}$ exists, it is unique and it has a chart expression given by

$$
\alpha_{\varepsilon}=\varepsilon_{i} \omega^{i} \wedge d t+\frac{1}{4}\left(\frac{\partial \varepsilon_{i}}{\partial \dot{x}^{j}}-\frac{\partial \varepsilon_{j}}{\partial \dot{x}^{i}}\right) \omega^{i} \wedge \omega^{j}+\frac{\partial \varepsilon_{i}}{\partial \ddot{x}^{j}} \omega^{i} \wedge \dot{\omega}^{j} .
$$

The identities (2.4), or equivalently (2.6) -(2.8), are called the Helmholtz conditions of local variationality (cf. Krupková and Prince [10, and references therein). Formula (2.10) yields the Vainberg-Tonti Lagrange function for a locally variational source form (see Tonti [14, Krupka [5]), which can always be reduced to first-order Lagrange function (2.9).

Remark 2. The Euler-Lagrange form $E_{\lambda}$ (2.3), associated with the Vainberg-Tonti Lagrangian $\lambda=\mathscr{L} d t$ (2.10), coincides with source form $\varepsilon$, provided the Helmholtz conditions (2.4) are satisfied. We also note that Helmholtz conditions (2.4) yield the following identity

$$
\frac{\partial B_{i k}}{\partial x^{j}}-\frac{\partial B_{j k}}{\partial x^{i}}-\frac{1}{2} \frac{\partial}{\partial \dot{x}^{k}}\left(\frac{\partial A_{i}}{\partial \dot{x}^{j}}-\frac{\partial A_{j}}{\partial \dot{x}^{i}}\right)=0,
$$


which is, however, dependent on Helmholtz conditions on $A_{i}, B_{i j}$, 2.6 2.8).

A 2 -form $\alpha$ on $\mathbb{R} \times T M$ is called a Lepage 2-form, if $\alpha$ is closed and admits a decomposition $\left(\pi^{2,1}\right)^{*} \alpha=E+F$, where $E$ is $\pi^{2,0}$-horizontal 1-contact 2-form on $\mathbb{R} \times T^{2} M$, and $F$ is a 2-contact 2 -form on $\mathbb{R} \times T^{2} M$. 2-form $\alpha_{\varepsilon}$, described by Theorem 1 (f), (2.11), is a Lepage 2 -form, called the Lepage equivalent of a source form $\varepsilon$.

The notion of a Lepage 2-form in fibered mechanics is due to Krupková 8] (see also generalizations by Krupková and Prince [9, 10]), and it contributes to the theory of Lepage forms introduced by Krupka [5, and references therein. For further application, we point out the following result.

Theorem 3. Every second-order Lagrangian $\lambda$ on $\mathbb{R} \times T^{2} M$ has a unique Lepage equivalent $\Theta_{\lambda}$ on $\mathbb{R} \times T^{3} M$. In a fibered chart on $\mathbb{R} \times M$, if $\lambda=\mathscr{L} d t$, then $\Theta_{\lambda}$ has the expression

$$
\Theta_{\lambda}=\mathscr{L} d t+\left(\frac{\partial \mathscr{L}}{\partial \dot{x}^{i}}-\frac{d}{d t} \frac{\partial \mathscr{L}}{\partial \ddot{x}^{i}}\right) \omega^{i}+\frac{\partial \mathscr{L}}{\partial \ddot{x}^{i}} \dot{\omega}^{i} .
$$

Moreover, a source form $\varepsilon$ on $\mathbb{R} \times T^{2} M$ is globally variational if and only if the equation

$$
\left(\pi^{2,1}\right)^{*} \alpha_{\varepsilon}=d \Theta_{\lambda}
$$

has a global solution $\lambda$ on $\mathbb{R} \times T^{2} M$.

Remark 4. $\Theta_{\lambda}$ (2.13) is the well-known Cartan form in Lagrangian mechanics; cf. Krupka, Krupková and Saunders 3]. Since $\Theta_{\lambda}$ is the Lepage equivalent of Lagrangian $\lambda$, it satisfies $p_{1} d \Theta_{\lambda}=E_{\lambda}$. We also point out $\Theta_{\lambda}$ depends on the choice of a Lagrangian $\lambda$ whereas $d \Theta_{\lambda}$ does not; $d \Theta_{\lambda}$ is decomposable as $d \Theta_{\lambda}=E_{\lambda}+F$, where $E_{\lambda}$ (2.3) is the Euler-Lagrange form of $\lambda$, and $F$ is a 2-contact 2-form.

\section{Exactness equation for Lepage 2 -Forms on $\mathbb{R} \times T^{2} M$}

Let $\varepsilon$ be a locally variational source form on $\mathbb{R} \times T^{2} M$, and $\alpha_{\varepsilon}$ be the Lepage equivalent of $\varepsilon$ (Theorem 11. (f), (2.11)). Since $\alpha_{\varepsilon}$ is closed, it is also locally exact according to the Poincaré lemma. In this section, we study the exactness equation for Lepage 2-form $\alpha_{\varepsilon}$ globally, with the aim to construct a global Lagrangian, provided $\varepsilon$ is in addition globally variational. That is, we search for a solution $\mu$ on $\mathbb{R} \times T M$ of the equation

$$
\alpha_{\varepsilon}=d \mu .
$$

Clearly, equation (3.1) need not have a solution, and even if solvability of (3.1) is assured, no general construction of its solution is known.

Properties of the Cartan equivalent $\Theta_{\lambda}$ of a global Lagrangian $\lambda$ imply the following straightforward observation.

Lemma 5. Let $\alpha_{\varepsilon}$ be the Lepage equivalent of a globally variational source form $\varepsilon$. Suppose that a 1-form $\mu$ on $\mathbb{R} \times T M$ is a solution of (3.1). Then the horizontal component $h \mu$ of $\mu$ is a Lagrangian on $\mathbb{R} \times T^{2} M$ for $\varepsilon$.

Proof. Since the Cartan equivalent $\Theta_{\lambda}$ of a global Lagrangian $\lambda$ for $\varepsilon$ obeys the property $\alpha_{\varepsilon}=d \Theta_{\lambda}$, we obtain $\mu=\Theta_{\lambda}+d f$ for some function $f$ hence $h \mu=\lambda+h(d f)$. Thus, $h \mu$ and $\lambda$ are equivalent Lagrangians for $\varepsilon$, whose Lagrange functions differ by means of total derivative of $f$. 
The next lemma describes a global decomposition of $\alpha_{\varepsilon}$ into closed forms.

Lemma 6. Let $\alpha_{\varepsilon}$ be the Lepage equivalent of a locally variational source form $\varepsilon$ on $\mathbb{R} \times T^{2} M$. Then there is a unique decomposition of $\alpha_{\varepsilon}$ on $\mathbb{R} \times T M$,

$$
\alpha_{\varepsilon}=\alpha_{0} \wedge d t+\alpha^{\prime}
$$

where $\alpha_{0}$ and $\alpha^{\prime}$ are closed forms defined on TM. With respect to a chart $(V, \psi)$, $\psi=\left(x^{i}\right)$, on $M$, we have

$$
\alpha_{0}=\left(A_{i}-\frac{1}{2}\left(\frac{\partial A_{i}}{\partial \dot{x}^{j}}-\frac{\partial A_{j}}{\partial \dot{x}^{i}}\right) \dot{x}^{j}\right) d x^{i}+B_{i j} \dot{x}^{j} d \dot{x}^{i},
$$

and

$$
\alpha^{\prime}=\frac{1}{4}\left(\frac{\partial A_{i}}{\partial \dot{x}^{j}}-\frac{\partial A_{j}}{\partial \dot{x}^{i}}\right) d x^{i} \wedge d x^{j}+B_{i j} d x^{i} \wedge d \dot{x}^{j} .
$$

Proof. In every chart $(V, \psi), \psi=\left(x^{i}\right)$, on $M$, it is straightforward to verify that decomposition of $\alpha_{\varepsilon}$ (3.2) holds for $\alpha_{0}$ and $\alpha^{\prime}$, given by formulas (3.3) and (3.4). Since the Lepage equivalent $\alpha_{\varepsilon}$ of $\varepsilon$ is closed, and $\alpha_{0}, \alpha^{\prime}$ do not contain $d t$, it easily follows that both $\alpha_{0}, \alpha^{\prime}$ must be closed. To verify that for instance $d \alpha^{\prime}$ vanishes, we can also proceed directly with the help of Helmholtz conditions (2.6)-2.8) and (2.12).

It remains to show that the 2 -forms $\alpha_{0}, \alpha^{\prime}$ are (globally) defined on the tangent bundle $T M$. Since $\alpha_{\varepsilon}$ is globally defined on $\mathbb{R} \times T M$, it is sufficient to show that $\alpha^{\prime}$ is defined on $T M$. For an arbitrary coordinate transformation $x^{i}=x^{i}\left(\bar{x}^{j}\right)$ on $M$, we get the following identities,

$$
\begin{aligned}
& A_{i}=\bar{A}_{k} \frac{\partial \bar{x}^{k}}{\partial x^{i}}+\bar{B}_{k l} \frac{\partial \bar{x}^{k}}{\partial x^{i}} \frac{\partial^{2} \bar{x}^{l}}{\partial x^{p} x^{q}} \dot{x}^{p} \dot{x}^{q}, \\
& B_{i j}=\bar{B}_{k l} \frac{\partial \bar{x}^{k}}{\partial x^{i}} \frac{\partial \bar{x}^{l}}{\partial x^{j}} .
\end{aligned}
$$

Differentiating (3.5) we obtain with the help of the Helmholtz condition (2.6),

$$
\begin{aligned}
& \frac{\partial A_{i}}{\partial \dot{x}^{j}}-\frac{\partial A_{j}}{\partial \dot{x}^{i}} \\
& =\frac{\partial \bar{A}_{k}}{\partial \dot{\bar{x}}^{l}}\left(\frac{\partial \bar{x}^{l}}{\partial x^{j}} \frac{\partial \bar{x}^{k}}{\partial x^{i}}-\frac{\partial \bar{x}^{l}}{\partial x^{i}} \frac{\partial \bar{x}^{k}}{\partial x^{j}}\right)+2 \bar{B}_{k l}\left(\frac{\partial \bar{x}^{k}}{\partial x^{i}} \frac{\partial^{2} \bar{x}^{l}}{\partial x^{j} x^{p}}-\frac{\partial \bar{x}^{k}}{\partial x^{j}} \frac{\partial^{2} \bar{x}^{l}}{\partial x^{i} x^{p}}\right) \dot{x}^{p} .
\end{aligned}
$$

From (3.6), (3.7), and using the transformation formulas

$$
\frac{\partial x^{i}}{\partial \bar{x}^{p}} \frac{\partial \bar{x}^{p}}{\partial x^{j}}=\delta_{j}^{i}, \quad \frac{\partial^{2} \bar{x}^{l}}{\partial x^{i} x^{j}} \frac{\partial x^{i}}{\partial \bar{x}^{p}} \frac{\partial x^{j}}{\partial \bar{x}^{q}}=-\frac{\partial \bar{x}^{l}}{\partial x^{j}} \frac{\partial^{2} x^{j}}{\partial \bar{x}^{p} \partial \bar{x}^{q}},
$$

we now obtain

$$
\begin{aligned}
& \frac{1}{4}\left(\frac{\partial A_{i}}{\partial \dot{x}^{j}}-\frac{\partial A_{j}}{\partial \dot{x}^{i}}\right) d x^{i} \wedge d x^{j}+B_{i j} d x^{i} \wedge d \dot{x}^{j} \\
& =\frac{1}{4}\left(\frac{\partial \bar{A}_{k}}{\partial \dot{\bar{x}}^{l}}-\frac{\partial \bar{A}_{l}}{\partial \dot{\bar{x}}^{k}}\right) d \bar{x}^{k} \wedge d \bar{x}^{l}+\bar{B}_{k l} \frac{\partial^{2} \bar{x}^{l}}{\partial x^{i} x^{j}} \frac{\partial x^{i}}{\partial \bar{x}^{p}} \frac{\partial x^{j}}{\partial \bar{x}^{q}} \dot{\bar{x}}^{q} d \bar{x}^{k} \wedge d \bar{x}^{p} \\
& +\bar{B}_{k l} \frac{\partial \bar{x}^{l}}{\partial x^{j}} \frac{\partial^{2} x^{j}}{\partial \bar{x}^{p} \partial \bar{x}^{q}} \dot{\bar{x}}^{p} d \bar{x}^{k} \wedge d \bar{x}^{q}+\bar{B}_{k l} \frac{\partial \bar{x}^{l}}{\partial x^{j}} \frac{\partial x^{j}}{\partial \bar{x}^{q}} d \bar{x}^{k} \wedge d \dot{\bar{x}}^{q} \\
& =\frac{1}{4}\left(\frac{\partial \bar{A}_{k}}{\partial \dot{\bar{x}}^{l}}-\frac{\partial \bar{A}_{l}}{\partial \dot{\bar{x}}^{k}}\right) d \bar{x}^{k} \wedge d \bar{x}^{l}+\bar{B}_{k l} d \bar{x}^{k} \wedge d \dot{\bar{x}}^{l}
\end{aligned}
$$

as required. 
Lemma 7. The equation

$$
\alpha_{0} \wedge d t=d \mu_{0}
$$

has always a solution $\mu_{0}=-t \alpha_{0}$ defined on $\mathbb{R} \times T M$. With respect to a chart $(V, \psi), \psi=\left(x^{i}\right)$, on $M, \mu_{0}$ is expressed as

$$
\mu_{0}=-\left(A_{i}-\frac{1}{2}\left(\frac{\partial A_{i}}{\partial \dot{x}^{j}}-\frac{\partial A_{j}}{\partial \dot{x}^{i}}\right) \dot{x}^{j}\right) t d x^{i}-B_{i j} \dot{x}^{i} t d \dot{x}^{j},
$$

and the horizontal component $h \mu_{0}$ of $\mu_{0}$, defined on $\mathbb{R} \times T^{2} M$, reads

$$
h \mu_{0}=-\varepsilon_{i} \dot{x}^{i} t d t
$$

where $\varepsilon_{i}=A_{i}+B_{i j} \ddot{x}^{j}$, see (2.5).

Proof. From Lemma 6 it follows that the 2 -form $\mu_{0}=-t \alpha_{0}$ is globally defined on $\mathbb{R} \times T M$, and a straightforward calculation shows that $\mu_{0}$ solves equation (3.8). The expression (3.10) can be then easily obtained in a chart by applying the horizontal morphism $h$ to the expression (3.9).

We now study the equation

$$
\alpha^{\prime}=d \mu^{\prime},
$$

where $\alpha^{\prime}$ is given by formula (3.4). To this purpose we define canonical local sections and homotopy operators as follows.

Let $(V, \psi), \psi=\left(x^{i}\right)$, be a fixed chart on $M$, and $\left(V^{1}, \psi^{1}\right), \psi^{1}=\left(x^{i}, \dot{x}^{i}\right)$, be the associated chart on $T M$. We put for every $l, 1 \leq l \leq m$,

$$
\pi_{l}^{1}\left(x^{i}, \dot{x}^{l}, \dot{x}^{k}\right)=\left(x^{i}, \dot{x}^{k}\right), \quad 1 \leq i \leq m, \quad l+1 \leq k \leq m,
$$

and

$$
s_{l, \nu_{l}}^{1}\left(x^{i}, \dot{x}^{k}\right)=\left(x^{i}, \nu_{l}, \dot{x}^{k}\right), \quad 1 \leq i \leq m, \quad l+1 \leq k \leq m .
$$

Local projections $\pi_{l}^{1}$, defined by formula (3.11), map open subsets $V_{l}^{1}$ of the chart domain $V^{1}$, described by equations $\dot{x}^{j}=0,1 \leq j \leq l-1$, onto $V_{l+1}^{1}$, whereas local sections $s_{l, \nu_{l}}^{1}$ (3.12) of $\pi_{l}^{1}$ map $V_{l+1}^{1}$ into $V_{l}^{1}$ for every $l, 1 \leq l \leq m$. Note that in this notation $V_{1}^{1}=V^{1}, V_{m+1}^{1}=V$ are the chart domains in $T M$ and $M$, respectively.

Define local homotopy operators as follows. For every $l, 1 \leq l \leq m$, let $K_{l}$ acts on (local) differential forms defined on $V_{l}^{1} \subset V^{1}$ by the formula

$$
K_{l} \rho=\int_{0}^{\dot{x}^{l}}\left(\pi_{l}^{1}\right)^{*}\left(s_{l, \nu_{l}}^{1}\right)^{*}\left(i_{\frac{\partial}{\partial \dot{x}^{l}}} \rho\right) d \nu^{l},
$$

where $\pi_{l}^{1}$ and $s_{l, \nu_{l}}^{1}$ are given by (3.11) and (3.12), and the integration operation in (3.13) is applied on coefficients of the corresponding differential form.

Theorem 8. Let $\alpha_{\varepsilon}$ be the Lepage equivalent of $\varepsilon$, and let $\alpha^{\prime}$ be the uniquelly given 2 -form by means of the decomposition (3.2), with local expression (3.4). Then

$$
\alpha^{\prime}-\omega=d \kappa,
$$

where

$$
\begin{aligned}
\omega & =\left(s_{1,0}^{1} \circ s_{2,0}^{1} \circ \ldots \circ s_{m, 0}^{1} \circ \pi_{m}^{1} \circ \pi_{m-1}^{1} \circ \ldots \circ \pi_{1}^{1}\right)^{*} \alpha^{\prime} \\
& =\frac{1}{4}\left(\frac{\partial A_{i}}{\partial \dot{x}^{j}}-\frac{\partial A_{j}}{\partial \dot{x}^{i}}\right)_{\left(x^{p}, 0\right)} d x^{i} \wedge d x^{j}
\end{aligned}
$$


and

$$
\begin{aligned}
\kappa & =\sum_{l=1}^{m}\left(\pi_{1}^{1}\right)^{*}\left(\pi_{2}^{1}\right)^{*} \ldots\left(\pi_{l-1}^{1}\right)^{*} K_{l}\left(\left(s_{l-1,0}^{1}\right)^{*} \ldots\left(s_{2,0}^{1}\right)^{*}\left(s_{1,0}^{1}\right)^{*} \alpha^{\prime}\right) \\
& =-\sum_{l=1}^{m} \int_{0}^{\dot{x}^{l}} B_{j l}\left(x^{p}, 0, \ldots, 0, \nu_{(l)}, \dot{x}^{l+1}, \ldots, \dot{x}^{m}\right) d \nu_{(l)} \cdot d x^{j}
\end{aligned}
$$

Proof. First, we prove the identity

$$
\alpha^{\prime}-\left(\pi_{1}^{1}\right)^{*}\left(s_{1,0}^{1}\right)^{*} \alpha^{\prime}=d\left(K_{1} \alpha^{\prime}\right)
$$

Using the chart expression of $\alpha^{\prime}$ (3.4), we get the left-hand side of (3.17) as

$$
\begin{aligned}
& \alpha^{\prime}-\left(\pi_{1}^{1}\right)^{*}\left(s_{1,0}^{1}\right)^{*} \alpha^{\prime} \\
& =\frac{1}{4}\left(\frac{\partial A_{i}}{\partial \dot{x}^{j}}-\frac{\partial A_{j}}{\partial \dot{x}^{i}}\right) d x^{i} \wedge d x^{j}+B_{i j} d x^{i} \wedge d \dot{x}^{j} \\
& -\frac{1}{4}\left(\frac{\partial A_{i}}{\partial \dot{x}^{j}}-\frac{\partial A_{j}}{\partial \dot{x}^{i}}\right)_{\left(x^{p}, 0, \dot{x}^{2}, \ldots, \dot{x}^{m}\right)} d x^{i} \wedge d x^{j}-\sum_{j=2}^{m} B_{i j}\left(x^{p}, 0, \dot{x}^{2}, \ldots, \dot{x}^{m}\right) d x^{i} \wedge d \dot{x}^{j} .
\end{aligned}
$$

Using the definition $K_{l}$ (3.13), we have $i_{\partial / \partial \dot{x}^{1}} \alpha^{\prime}=-B_{1 i} d x^{i}$ and the right-hand side of (3.17) reads

$$
\begin{aligned}
& d\left(K_{1} \alpha^{\prime}\right) \\
& =d\left(\int_{0}^{\dot{x}^{1}}\left(\pi_{1}^{1}\right)^{*}\left(s_{1, \nu}^{1}\right)^{*}\left(i_{\frac{\partial}{\partial \dot{x}^{1}}} \alpha^{\prime}\right) d \nu\right)=-d\left(\int_{0}^{\dot{x}^{1}} B_{1 i}\left(x^{p}, \nu, \dot{x}^{2}, \ldots, \dot{x}^{m}\right) d \nu\right) \wedge d x^{i} \\
& =\frac{1}{2}\left(\int_{0}^{\dot{x}^{1}}\left(\frac{\partial B_{1 i}}{\partial x^{j}}-\frac{\partial B_{1 j}}{\partial x^{i}}\right)_{\left(x^{p}, \nu, \dot{x}^{2}, \ldots, \dot{x}^{m}\right)} d \nu\right) d x^{i} \wedge d x^{j} \\
& +B_{1 i} d x^{i} \wedge d \dot{x}^{1}-\sum_{j=2}^{m}\left(\int_{0}^{\dot{x}^{1}}\left(\frac{\partial B_{1 i}}{\partial \dot{x}^{j}}\right)_{\left(x^{p}, \nu, \dot{x}^{2}, \ldots, \dot{x}^{m}\right)} d \nu\right) d \dot{x}^{j} \wedge d x^{i} .
\end{aligned}
$$

We now apply the Helmholtz conditions (2.6) and (2.12),

$$
B_{i j}=B_{j i}, \quad \frac{\partial B_{i k}}{\partial \dot{x}^{j}}=\frac{\partial B_{j k}}{\partial \dot{x}^{i}}, \quad \frac{\partial B_{i k}}{\partial x^{j}}-\frac{\partial B_{j k}}{\partial x^{i}}=\frac{1}{2} \frac{\partial}{\partial \dot{x}^{k}}\left(\frac{\partial A_{i}}{\partial \dot{x}^{j}}-\frac{\partial A_{j}}{\partial \dot{x}^{i}}\right)
$$


and obtain

$$
\begin{aligned}
d & \left(K_{1} \alpha^{\prime}\right) \\
= & \frac{1}{4}\left(\int_{0}^{\dot{x}^{1}} \frac{\partial}{\partial \dot{x}^{1}}\left(\frac{\partial A_{i}}{\partial \dot{x}^{j}}-\frac{\partial A_{j}}{\partial \dot{x}^{i}}\right)_{\left(x^{p}, \nu, \dot{x}^{2}, \ldots, \dot{x}^{m}\right)} d \nu\right) d x^{i} \wedge d x^{j} \\
& +B_{1 i} d x^{i} \wedge d \dot{x}^{1}-\sum_{j=2}^{m}\left(\int_{0}^{\dot{x}^{1}}\left(\frac{\partial B_{i j}}{\partial \dot{x}^{1}}\right)_{\left(x^{p}, \nu, \dot{x}^{2}, \ldots, \dot{x}^{m}\right)} d \nu\right) d \dot{x}^{j} \wedge d x^{i} \\
= & \frac{1}{4}\left(\left(\frac{\partial A_{i}}{\partial \dot{x}^{j}}-\frac{\partial A_{j}}{\partial \dot{x}^{i}}\right)-\left(\frac{\partial A_{i}}{\partial \dot{x}^{j}}-\frac{\partial A_{j}}{\partial \dot{x}^{i}}\right)_{\left(x^{p}, 0, \dot{x}^{2}, \ldots, \dot{x}^{m}\right)}\right) d x^{i} \wedge d x^{j} \\
& +B_{i 1} d x^{i} \wedge d \dot{x}^{1}+\sum_{j=2}^{m}\left(B_{i j}-B_{i j}\left(x^{p}, 0, \dot{x}^{2}, \ldots, \dot{x}^{m}\right)\right) d x^{i} \wedge d \dot{x}^{j}, \\
= & \frac{1}{4}\left(\frac{\partial A_{i}}{\partial \dot{x}^{j}}-\frac{\partial A_{j}}{\partial \dot{x}^{i}}\right)^{d} \wedge d x^{i} \wedge \frac{1}{4}\left(\frac{\partial A_{i}}{\partial \dot{x}^{j}}-\frac{\partial A_{j}}{\partial \dot{x}^{i}}\right)_{\left(x^{p}, 0, \dot{x}^{2}, \ldots, \dot{x}^{m}\right)} d x^{i} \wedge d x^{j} \\
& +B_{i j} d x^{i} \wedge d \dot{x}^{j}-\sum_{j=2}^{m} B_{i j}\left(x^{p}, 0, \dot{x}^{2}, \ldots, \dot{x}^{m}\right) d x^{i} \wedge d \dot{x}^{j},
\end{aligned}
$$

as required to show (3.17). By means of similar arguments we observe that the following formula holds

$$
\begin{aligned}
& \left(s_{l-1,0}^{1}\right)^{*} \ldots\left(s_{1,0}^{1}\right)^{*} \alpha^{\prime} \\
& \quad=\left(\pi_{l}^{1}\right)^{*}\left(s_{l, 0}^{1}\right)^{*}\left(s_{l-1,0}^{1}\right)^{*} \ldots\left(s_{1,0}^{1}\right)^{*} \alpha^{\prime}+d\left(K_{l}\left(\left(s_{l-1,0}^{1}\right)^{*} \ldots\left(s_{1,0}^{1}\right)^{*} \alpha^{\prime}\right)\right)
\end{aligned}
$$

for every $l, 1 \leq l \leq m$. Applying formula (3.18) recurrently, we now easily obtain

$$
\begin{aligned}
& \alpha^{\prime}=\left(\pi_{1}^{1}\right)^{*}\left(\pi_{2}^{1}\right)^{*} \ldots\left(\pi_{m}^{1}\right)^{*}\left(s_{m, 0}^{1}\right)^{*}\left(s_{m-1,0}^{1}\right)^{*} \ldots\left(s_{1,0}^{1}\right)^{*} \alpha^{\prime} \\
& +d\left(\sum_{l=1}^{m}\left(\pi_{1}^{1}\right)^{*}\left(\pi_{2}^{1}\right)^{*} \ldots\left(\pi_{l-1}^{1}\right)^{*} K_{l}\left(\left(s_{l-1,0}^{1}\right)^{*} \ldots\left(s_{2,0}^{1}\right)^{*}\left(s_{1,0}^{1}\right)^{*} \alpha^{\prime}\right)\right),
\end{aligned}
$$

as required.

The identity (3.14) of Theorem 8 is formulated by means charts. We now show that (3.14) is a global decomposition of $\alpha^{\prime}$.

Theorem 9. Both $\kappa$ (3.16) and $\omega$ (3.15) define (global) differential 1-forms on $T M$.

Proof. We prove that the local expressions for both $\omega$ (3.15) and $\kappa$ (3.16) coincide on the intersection of two overlapping charts on $T M$. To this purpose let $\bar{\Psi} \circ$ $\Psi^{-1}\left(x^{i}, \dot{x}^{i}\right)=\left(\bar{x}^{j}, \dot{\bar{x}}^{j}\right)$ be the coordinate transformation between charts $(V, \psi)$, $\psi=\left(x^{i}, \dot{x}^{i}\right)$, and $(\bar{V}, \bar{\psi}), \bar{\psi}=\left(\bar{x}^{i}, \dot{\bar{x}}^{i}\right)$, on $T M$, where $\bar{x}^{j}=\bar{x}^{j}\left(x^{i}\right)$ and $\dot{\bar{x}}^{j}=$ $\dot{\bar{x}}^{j}\left(x^{i}, \dot{x}^{i}\right)$.

1. From (3.7) we have

$$
\left(\frac{\partial A_{i}}{\partial \dot{x}^{j}}-\frac{\partial A_{j}}{\partial \dot{x}^{i}}\right)_{\left(x^{p}, 0\right)}=\left(\frac{\partial \bar{A}_{k}}{\partial \dot{\bar{x}}^{l}}-\frac{\partial \bar{A}_{l}}{\partial \dot{\bar{x}}^{k}}\right)_{\left(\bar{x}^{p}, 0\right)} \frac{\partial \bar{x}^{l}}{\partial x^{j}} \frac{\partial \bar{x}^{k}}{\partial x^{i}}
$$


hence the transformation of the local formula (3.15) for $\omega$ reads

$$
\begin{aligned}
& \omega=\frac{1}{4}\left(\frac{\partial A_{i}}{\partial \dot{x}^{j}}-\frac{\partial A_{j}}{\partial \dot{x}^{i}}\right)_{\left(x^{p}, 0\right)} d x^{i} \wedge d x^{j} \\
& =\frac{1}{4}\left(\frac{\partial \bar{A}_{k}}{\partial \dot{\bar{x}}^{l}}-\frac{\partial \bar{A}_{l}}{\partial \dot{\bar{x}}^{k}}\right)_{\left(\bar{x}^{p}, 0\right)} \frac{\partial \bar{x}^{l}}{\partial x^{j}} \frac{\partial \bar{x}^{k}}{\partial x^{i}} \frac{\partial x^{i}}{\partial \bar{x}^{u}} \frac{\partial x^{j}}{\partial \bar{x}^{v}} d \bar{x}^{u} \wedge d \bar{x}^{v} \\
& =\frac{1}{4}\left(\frac{\partial \bar{A}_{k}}{\partial \dot{\bar{x}}^{l}}-\frac{\partial \bar{A}_{l}}{\partial \dot{\bar{x}}^{k}}\right)_{\left(\bar{x}^{p}, 0\right)} d \bar{x}^{k} \wedge d \bar{x}^{l},
\end{aligned}
$$

as required.

2. Consider the local expression for $\kappa$ (3.16) to which we apply the change of variables theorem for integrals of differential forms. Employing the corresponding transformation properties described for every $l, 1 \leq l \leq m$, by

$$
\bar{\Psi} \circ \Psi^{-1}\left(x^{i},\left(0, \ldots 0, \nu_{(l)}, \dot{x}^{l+1}, \ldots, \dot{x}^{m}\right)\right)=\left(\bar{x}^{j}, \bar{\mu}_{(l)}^{j}\right),
$$

where

$$
\bar{\mu}_{(l)}^{q}=\frac{\partial \bar{x}^{q}}{\partial x^{l}} \nu_{(l)}+\sum_{k=l+1}^{m} \frac{\partial \bar{x}^{q}}{\partial x^{k}} \dot{x}^{k}, \quad 1 \leq q \leq m,
$$

and for $1 \leq s \leq l-1, l+1 \leq k \leq m$,

$$
0=\frac{\partial x^{s}}{\partial \bar{x}^{j}} \bar{\mu}_{(l)}^{j}, \quad \nu_{(l)}=\frac{\partial x^{l}}{\partial \bar{x}^{j}} \bar{\mu}_{(l)}^{j}, \quad \dot{x}^{k}=\frac{\partial x^{k}}{\partial \bar{x}^{j}} \bar{\mu}_{(l)}^{j},
$$

we obtain using (3.6) the coordinate transformation for $\kappa(3.16)$, where the integrals over segments are transformed into line integrals,

$$
\begin{aligned}
& \kappa=-\sum_{l=1}^{m} \int_{0}^{\dot{x}^{l}} B_{j l}\left(x^{p}, 0, \ldots, 0, \nu_{(l)}, \dot{x}^{l+1}, \ldots, \dot{x}^{m}\right) d \nu_{(l)} \cdot d x^{j} \\
& =-\sum_{l=1}^{m} \int_{\bar{\mu}_{(l)}^{p}=\sum_{k=l+1}^{m} \frac{\partial \bar{x}^{p} \dot{x}^{k}}{\partial x^{k}}}^{\bar{x}_{(l)}^{p}=\sum_{k=l}^{m} \frac{\partial \bar{x}^{p} \dot{x}^{k}}{\partial x^{k}}} \bar{B}_{u v}\left(\bar{x}^{i}, \bar{\mu}_{(l)}^{i}\right) \frac{\partial \bar{x}^{u}}{\partial x^{j}} \frac{\partial \bar{x}^{v}}{\partial x^{l}} \frac{\partial x^{j}}{\partial \bar{x}^{w}} \frac{\partial x^{l}}{\partial \bar{x}^{q}} d \bar{\mu}_{(l)}^{q} \cdot d \bar{x}^{w} \\
& =-\sum_{l=1}^{m} \int_{\bar{\mu}_{(l)}^{p}=\sum_{k=l+1}^{m}=\sum_{k=l}^{m} \frac{\partial \bar{x}^{p}}{\partial x^{k} \dot{x}^{k}}}^{\frac{\partial \bar{x}^{p} \dot{x}^{k}}{\partial x^{k}} \dot{x}^{k}} \bar{B}_{u v}\left(\bar{x}^{i}, \bar{\mu}_{(l)}^{i}\right) \frac{\partial \bar{x}^{v}}{\partial x^{l}} \frac{\partial x^{l}}{\partial \bar{x}^{q}} d \bar{\mu}_{(l)}^{q} \cdot d \bar{x}^{u} .
\end{aligned}
$$

Since the coordinate functions $x^{i}$ and $\dot{x}^{k}, l+1 \leq k \leq m$, are constant with respect to the integration in (3.20), from (3.19) we get

$$
\frac{\partial x^{j}}{\partial \bar{x}^{q}} d \bar{\mu}_{(l)}^{q}=0, \quad j \neq l,
$$

hence in (3.20) for every $l, 1 \leq l \leq m$,

$$
\frac{\partial \bar{x}^{v}}{\partial x^{l}} \frac{\partial x^{l}}{\partial \bar{x}^{q}} d \bar{\mu}_{(l)}^{q}=\sum_{j=1}^{m} \frac{\partial \bar{x}^{v}}{\partial x^{j}} \frac{\partial x^{j}}{\partial \bar{x}^{q}} d \bar{\mu}_{(l)}^{q}=\delta_{q}^{v} d \bar{\mu}_{(l)}^{q}=d \bar{\mu}_{(l)}^{v} .
$$


Using (3.21), formula (3.20) now reads

$$
\begin{aligned}
& \kappa=-\sum_{l=1}^{m} \int_{\bar{\mu}_{(l)}^{p}=\sum_{k=l+1}^{m} \frac{\partial \bar{x}^{p} \dot{\mu}^{k}}{\partial x^{k}} \bar{x}^{p}}^{\bar{B}_{(l)}^{p}=\sum_{k=l}^{m} \frac{\partial \bar{x}^{p}}{\partial x^{k}} \dot{x}^{k}}\left(\bar{x}^{i}, \bar{\mu}_{(l)}^{i}\right) d \bar{\mu}_{(l)}^{v} \cdot d \bar{x}^{u} \\
& =-\int_{\bar{\mu}^{p}=0}^{\bar{\mu}^{p}=\dot{\bar{x}}^{p}} \bar{B}_{u v}\left(\bar{x}^{i}, \bar{\mu}^{i}\right) d \bar{\mu}^{v} \cdot d \bar{x}^{u} .
\end{aligned}
$$

Since the functions $\bar{B}_{u v}=\bar{B}_{u v}\left(\bar{x}^{i}, \bar{\mu}^{i}\right)$ satisfy the Helmholtz condition (2.6),

$$
\frac{\partial \bar{B}_{u w}}{\partial \mu^{v}}=\frac{\partial \bar{B}_{v w}}{\partial \mu^{u}}
$$

the line integrals (3.22) for every $u, 1 \leq u \leq m$, are independent upon choice of a path connecting the points $(0,0, \ldots, 0)$ and $\left(\dot{\bar{x}}^{1}, \dot{\bar{x}}^{2}, \ldots, \dot{\bar{x}}^{m}\right)$. Thus, in (3.22) we are allowed to integrate over segments on an $m$-dimensional cube, that is

$$
\begin{aligned}
\kappa & =-\int_{\bar{\mu}^{p}=0}^{\bar{\mu}^{p} \overline{\bar{x}}^{p}} \bar{B}_{u v}\left(\bar{x}^{i}, \bar{\mu}^{i}\right) d \bar{\mu}^{v} \cdot d \bar{x}^{u} \\
& =-\sum_{v=1}^{m} \int_{0}^{\dot{\bar{x}}^{v}} \bar{B}_{u v}\left(\bar{x}^{i}, 0, \ldots, 0, \bar{\mu}^{v}, \dot{\bar{x}}^{v+1}, \ldots, \dot{\bar{x}}^{m}\right) d \bar{\mu}^{v} \cdot d \bar{x}^{u},
\end{aligned}
$$

proving that (3.16) defines global differential 1-form $\kappa$ on $T M$.

Theorem 9 implies that $\alpha^{\prime}=\omega+d \kappa$ is globally defined. Using this fact and applying Lemma 6 and 7, we get a global decomposition of $\alpha_{\varepsilon}$,

$$
\alpha_{\varepsilon}=\omega+d\left(\mu_{0}+\kappa\right) .
$$

Clearly, the problem of global exactness of the Lepage equivalent $\alpha_{\varepsilon}$ is by means of (3.23) reduced to global exactness of 2 -form $\omega$ (3.15) defined on $M$. In other words, if $\omega$ is globally exact, then so is $\alpha_{\varepsilon}$ hence the source form $\varepsilon$ is globally variational.

Remark 10. In general, if $M$ is an $m$-dimensional smooth manifold and $\rho$ is a closed differential $k$-form on $M, k \leq m$, then the equation $\rho=d \mu$ need not have a (global) solution $\mu$ on $M$. Indeed, it is the $k$-th de Rham cohomology group $H_{\mathrm{dR}}^{k} M=\operatorname{Ker} d_{k} / \operatorname{Im} d_{k-1}$ which decides about solvability of the exactness equation $\rho=d \mu$. If $H_{\mathrm{dR}}^{k} M$ is trivial, then $\rho=d \mu$ has always a solution $\mu$ on $M$. Nevertheless, in this case $\left(H_{\mathrm{dR}}^{k} M=0\right)$ there is no general constructive procedure of finding a solution $\eta$ for a given closed $k$-form $\rho$, where $k<m$; if $k=m$, to find a solution one can apply the top-cohomology theorems (cf. Lee [1]).

Corollary 11. If the 2-form $\omega$ (3.15) vanishes, i.e. the coefficients of $\omega$ satisfy

$$
\left(\frac{\partial A_{i}}{\partial \dot{x}^{j}}-\frac{\partial A_{j}}{\partial \dot{x}^{i}}\right)_{\left(x^{p}, 0\right)}=0
$$

in every chart, then source form $\varepsilon$ is globally variational and it admits a Lagrangian on $\mathbb{R} \times T M$, namely

$$
\lambda=h\left(\mu_{0}+\kappa\right),
$$

where $\mu_{0}$ and $\kappa$ are given by (3.9) and (3.16), respectively. 
Proof. This is an immediate consequence of Theorems 8 and 9 and Lemmas 6 and 7. Indeed, using (3.23) we get

$$
\alpha_{\varepsilon}=\alpha_{0} \wedge d t+\alpha^{\prime}=d\left(\mu_{0}+\kappa\right),
$$

hence the horizontal part of $\mu_{0}+\kappa$ is a Lagrangian for $\varepsilon$; cf. Lemma 5 .

Remark $12(\operatorname{dim} M=2)$. In our paper [17, we studied the exactness equation for Lepage equivalents of source forms on $\mathbb{R} \times T^{2} M$, where $M$ is 2-dimensional connected smooth manifold. In the corresponding decomposition (3.23), the equation $d \eta=\omega$ is solvable and its solution $\eta$ can be constructed with the help of the topcohomology theorems. Examples of a global Lagrangian construction on concrete smooth 2-manifolds (Möbius strip, punctured torus) are also discussed.

\section{Globally variational homogeneous equations of Degree $c \neq 0,1$}

We briefly recall some basic facts on locally variational second-order ordinary differential equations, given by homogeneous functions of degree $c \neq 0,1$. More detailed exposition with proofs can be found in a recent paper by Rossi [12. Our main result consists in Theorem 14 showing that locally variational source forms with homogeneous coefficients of degree $c \neq 0,1$ are automatically globally variational.

A real-vauled function $F: T^{2} M \rightarrow \mathbb{R}$, resp. $F: T M \rightarrow \mathbb{R}$, is called homogeneous of degree $c$, if $F$ satisfies

$$
\frac{\partial F}{\partial \dot{x}^{i}} \dot{x}^{i}+2 \frac{\partial F}{\partial \ddot{x}^{i}} \ddot{x}^{i}=c F, \quad \text { resp. } \frac{\partial F}{\partial \dot{x}^{i}} \dot{x}^{i}=c F,
$$

with respect to any chart on $M$. Note that for $c=1, F: T M \rightarrow \mathbb{R}$ satisfying the Euler condition (4.1) is called a positive-homogenous function.

Let $\varepsilon$ be a locally variational source form $\mathbb{R} \times T^{2} M$. If the coefficients $\varepsilon_{i}=$ $A_{i}+B_{i j} \ddot{x}^{j}$ of $\varepsilon$ are homogeneous of degree $c \neq 0,1$, then using (4.1) it is readily seen that $A_{i}$ are homogeneous of degree $c$, and $B_{i j}$ are homogeneous of degree $c-2$, and vice versa. It is also straightforward to show that locally variational $\varepsilon_{i}$ are homogeneous of degree $c$ if and only if $\varepsilon_{i}$ possess a homogeneous Lagrangian of degree $c$.

The following theorem characterizes the structure of locally variational homogeneous source forms.

Theorem 13. Let $\varepsilon$ be a source form on $\mathbb{R} \times T^{2} M$, with coefficients homogeneous of degree $c \neq 0,1$, and affine in second derivatives, $\varepsilon_{i}=A_{i}+B_{i j} \ddot{x}^{j}$ (2.5). The following two conditions are equivalent:

(a) $\varepsilon$ is locally variational,

(b) functions $A_{i}, B_{i j}$, satisfy the subset of Helmholtz conditions (2.6)-(2.7),

$$
\begin{aligned}
& B_{i j}=B_{j i}, \quad \frac{\partial B_{i k}}{\partial \dot{x}^{j}}=\frac{\partial B_{j k}}{\partial \dot{x}^{i}}, \\
& \frac{\partial A_{i}}{\partial \dot{x}^{j}}+\frac{\partial A_{j}}{\partial \dot{x}^{i}}-2 \frac{\partial B_{i j}}{\partial x^{k}} \dot{x}^{k}=0 .
\end{aligned}
$$

Moreover, if $\varepsilon$ is locally variational, then $A_{i}$ satisfy

$$
A_{i}=\frac{1}{c-1}\left(\frac{1}{2}\left(\frac{\partial B_{i j}}{\partial x^{k}}+\frac{\partial B_{i k}}{\partial x^{j}}\right)-\frac{1}{c} \frac{\partial B_{j k}}{\partial x^{i}}\right) \dot{x}^{j} \dot{x}^{k} .
$$

Proof. See Rossi [12. 
Combining Theorem 13 with the results of Section 3, summarized by Corollary 11. we immediately obtain the following consequence for variational and homogeneous of degree $c \neq 0,1$ equations.

Theorem 14. Let $\varepsilon$ be a locally variational source form on $\mathbb{R} \times T^{2} M$, with coefficients homogeneous of degree $c \neq 0,1$. Then $\varepsilon$ is also globally variational, and it admits a global Lagrangian given by $\lambda=h\left(\mu_{0}+\kappa\right)$, where $\mu_{0}$ and $\kappa$ are 1 -forms on $\mathbb{R} \times T M$ given by (3.9) and (3.16), respectively.

Proof. From the assumptions on $\varepsilon_{i}=A_{i}+B_{i j} \ddot{x}^{j}$ it follows that $A_{i}$ has the expression given by Theorem 13. (4.2). Hence condition (3.24) holds, and by Corollary 11] $\varepsilon$ is globally variational, possessing a global Lagrangian $\lambda=h\left(\mu_{0}+\kappa\right)=\mathscr{L} d t$, where

$$
\mathscr{L}=-\varepsilon_{i} \dot{x}^{i} t-\sum_{l=1}^{m} \int_{0}^{\dot{x}^{l}} B_{j l}\left(x^{p}, 0, \ldots, 0, \nu_{(l)}, \dot{x}^{l+1}, \ldots, \dot{x}^{m}\right) d \nu_{(l)} \dot{x}^{j} .
$$

Remark 15. Standard examples of variational and homogeneous of degree 2 equations are, for instance, the geodesic equations in Riemann geometry, geodesic equations of a spray in Finsler geometry, as well as the geodesic equations of a Cartan connection (or metrizable connection, cf. Krupka and Sattarov [1]) on a tangent bundle, associated with a Finsler structure. Here, the functions $-B_{i j}$ are equal to components of metrics (Riemannian or Finsler), i.e. $B_{i j}=-g_{i j}$. Although it is straightforward from the nature of these equations arising from the (global) energy Lagrangian, we point out that global variationality of these systems follows from Theorem 14

\section{REFERENCES}

[1] D. Krupka and A. Sattarov, The inverse problem of the calculus of variations for Finsler structures, Math. Slovaca 35 (1985), 217-222.

[2] D. Krupka, Variational sequences in mechanics, Calc. Var. 5 (1997), 557-583.

[3] D. Krupka, O. Krupková, and D. Saunders, Cartan-Lepage forms in geometric mechanics, Int. J. Non-Linear Mechanics 47 (2012), 1154-1160.

[4] D. Krupka, Z. Urban, and J. Volná, Variational projectors in fibred manifolds, Miskolc Math. Notes 14, No. 2 (2013), 503-516.

[5] D. Krupka, Introduction to Global Variational Geometry, Atlantis Press, Amsterdam, 2015.

[6] D. Krupka, G. Moreno, Z. Urban, and J. Volná, On a bicomplex induced by the variational sequence, Int. J. Geom. Meth. Mod. Phys. 12, No. 5 (2015), 1550057.

[7] D. Krupka, Z. Urban, and J. Volná, Variational submanifolds of Euclidean spaces, J. Math. Phys. 59 (2018), 032903.

[8] O. Krupková, Lepagean 2-forms in higher order Hamiltonian mechanics, I. Regularity, Arch. Math. (Brno) 22 (1986) 97-120.

[9] O. Krupková and G.E. Prince, Lepage forms, closed 2-forms and second-order ordinary differential equations, Russian Mathematics 51, No. 12 (2007), 1-16.

[10] O. Krupková and G.E. Prince, Second order ordinary differential equations in jet bundles and the inverse problem of the calculus of variations, in: D. Krupka and D. Saunders (Eds.), Handbook of Global Analysis, Elsevier, Amsterdam, 2008, pp. 837-904.

[11] J.M. Lee, Introduction to Smooth Manifolds, GTM 218, Springer-Verlag, New York, 2002; 2nd Edition, 2012; Errata at: https://sites.math.washington.edu/ lee/Books/ISM/

[12] O. Rossi, Homogeneous differential equations and the inverse problem of the calculus of variations, Publ. Math. Debrecen 84, No. 1-2 (2014), 165-188.

[13] F. Takens, A global version of the inverse problem of the calculus of variations, J. Diff. Geom. 14 (1979), 543-562. 
[14] E. Tonti, Variational formulation of nonlinear differential equations I, Acad. Roy. Belg. Bull. Cl. Sci. 55 (1969), 137-165.

[15] Z. Urban and D. Krupka, The Zermelo conditions and higher order homogeneous functions, Publ. Math. Debrecen 82, No. 1 (2013), 59-76.

[16] Z. Urban and D. Krupka, The Helmholtz conditions for systems of second order homogeneous differential equations, Publ. Math. Debrecen 83, No. 1-2 (2013), 71-84.

[17] Z. Urban and J. Volná, On a global Lagrangian construction for ordinary variational equations on 2-manifolds, arXiv:1812.04270 [math.DG].

Z. Urban, Department of Mathematics, Faculty of Civil Engineering, VŠB-Technical University of Ostrava, Ludvíka Podéště 1875/17, 70833 Ostrava, Czech Republic

E-mail address: zbynek.urban@vsb.cz

J. Volná, Department of Mathematics, Faculty of Civil Engineering, VŠB-Technical University of Ostrava, Ludvíka Podéště 1875/17, 70833 Ostrava, Czech Republic

E-mail address: jana.volna@vsb.cz 